\title{
Intelligent Carpooling System
}

\author{
Anuja Shete \\ UG Student \\ Department of Computer \\ Engineering \\ Smt. Kashibai Navale College \\ of Engineering \\ Pune , India
}

\author{
Vedashree Bhandare \\ UG Student \\ Department of Computer \\ Engineering \\ Smt. Kashibai Navale College \\ of Engineering \\ Pune, India
}

\author{
Leena Londhe \\ UG Student \\ Department of Computer \\ Engineering \\ Smt. Kashibai Navale College \\ of Engineering \\ Pune, India
}

\author{
P.B.Mali \\ Professor \\ Department of Computer Engineering \\ Smt. Kashibai Navale College of Engineering \\ Pune, India
}

\begin{abstract}
The constant population and economic growth has caused an enormous increase in the number of private cars in cities worldwide. Carpooling is one of the most effective solutions to traffic congestion. In this paper, an advanced carpool system is described in detail and called the intelligent carpool system (ICS), which provides users the use of the carpool services through a smart handheld device anywhere and at any time. To generate ride matches through the carpool service agency, we use the genetic algorithm to propose the geneticbased carpool route and matching algorithm (GCRMA) for this multi objective optimization problem called the carpool service problem (CSP).Use of the GCRMA was proved to result in superior results involving the optimization objectives of CSP than other algorithms. Furthermore, our GCRMA has small amount of computational complexity to response the match results in the reasonable time, and the processing time is further reduced by the termination criteria of early stop. The remaining of this paper is organized as follows: Section I contains introduction to carpool system, Section II presents related search, Section III describes two modules of ICS, Section IV contains carpool system problem (CSP), while Section V contains genetic based algorithm i.e. GCRMA.
\end{abstract}

\section{Keywords}

Intelligent carpool system (ICS), genetic-based carpool route and matching algorithm (GCRMA), carpool service problem (CSP).

\section{INTRODUCTION}

Recent economic development has resulted in rapid increases in the number of vehicles on roadways and, thus, its causes serious traffic congestion problems in cities around the world. Severe traffic congestion can have many detrimental effects, such as time loss, air pollution, and increased fuel consumption [1], [2], [3]. However, each car usually transports just one or two individuals, resulting in many empty seats. For example, a vehicle in the U.K. is used to transport, on average, 1.5 people [4]. This represents an underuse of available transportation resources, a problem whose solution will require considerable effort. Carpooling is a relatively environmentally sound system;it consists of increasing the occupancy rate of cars by reducing the empty seats in these vehicles effectively. Drivers share their cars with one or more people who have similar transportation routes. Consequently, lesser vehicles will be needed to transport the same quantity of people to their respective destinations, resulting in substantially fewer cars on the road. Other carpooling benefits are reduced travel cost, energy consumption, and vehicle emissions. Due to technological advances such as the development of smart handheld device, along with mobile Internet technology, the web-based carpool system has become more advanced and is now referred as the intelligent carpool system (ICS). Through the use of smart handheld devices with Global Positioning System (GPS) navigation and mobile communication ability, users can instantaneously access real-time carpool service, with their current locations and other required information input by their smart phones, tablet, or other devices. Several such start-up systems, such as Carma, go2gether have been developed to coordinate ride-match communication between drivers and passengers in real-time carpool system.

\section{LITERATURE SURVEY}

Many projects have undertaken the carpooling problem and given various effective solutions to the problem. In distributed algorithm it was proposed that map the driver with the earliest departure time to his destination and one or more passengers through a path which has low cost that is minimum travel distance [5]. The process is repeated until the all the drivers have been allocate with passengers. This algorithm is simple and adapts easily to newcomers, but the solution is suboptimal, since the maximum percentage of picked up passengers was reported to be $80 \%$. The fairness component was also neglected in this solution. Another solution which is based on the Dijkstra Algorithm[6]. The network of users is divided into small areas centered on a driver. Each passenger is checked to see if a car with empty seats passes near him or her, if so then the assignment is performed incrementally. The runtime of this solution is fast as compared to other carpooling solutions, but the solution is not globally optimal, and thus it won't be fair when considering incremental driven distances. The Adaptive Genetic Algorithm is another way to solve the LTCPP. In this algorithm there is little knowledge about the search space [7]. The GA chromosome consists of pools of several users; half of the users are inserted using a greedy insertion method while the other half is inserted randomly. This approach has some drawback which are the individual fairness is sacrificed to reach a near-optimal solution and it is difficult to adapt to sudden changes in the system. 


\section{ICS}

Carpool services framework called the ICS, comprises two primary modules: a mobile clients (MC) module and a carpool services (CS) module. Communication can be established between the MC module and the CS module by using the web HTTP protocol through the mobile communication network.

\subsection{Module}

In order to give system users the opportunity to obtain carpool services anywhere and at any time, drivers and passengers can use the MC module to perform carpool operations through smart handled devices.The MC module is a mobile application built on an advanced mobile operating system such as Android. It uses GPS receiver. Therefore users can receive information about their current locations by automatically accessing the GPS signals of satellites. Using the MC module, driver can offer carpool rides and passenger can send carpool requests. The users have the option of rating their respective experiences with each other, which are visible to determine the potential of carpool service users.

\subsection{CS Module}

To support interoperability with the MC module, the CS module provides the RESTful webservice application interface for global implementation of the ICS on crossplatform devices. Google Cloud Messaging for Android is a service that allows you to send the data in form of a message from server to users' Android powered device, and vice versa. The GCM service handles the queuing of messages and delivery to the target Android application running on target device. GCM service is completely free. GCM Connection Servers receives messages from a 3rd-party app server in our case the CS module and send these messages to a GCMenabled client which corresponds to MC module. An app server sends the data in form of messages to a GCM connection server; the connection server collects and stores the message, and then sends it to the client. The Client App is a GCM-enabled. The app must register with GCM and get a registration ID.

\section{CSP}

CSP is a multi-purpose optimization problem since it simultaneously concerns more than one objective. Drivers travel to all source-destination pairings (pickup and drop-off locations) of assigned passengers who have made carpool request to the ICS system. The primary objective of the CSP is to match maximum number of passengers with drivers, as well as their cumulative credit score should be high. The secondary objective is to minimize the average distance travelled by drivers, the average waiting distance of passengers, and the average travel distance of passengers.

\section{GCRMA}

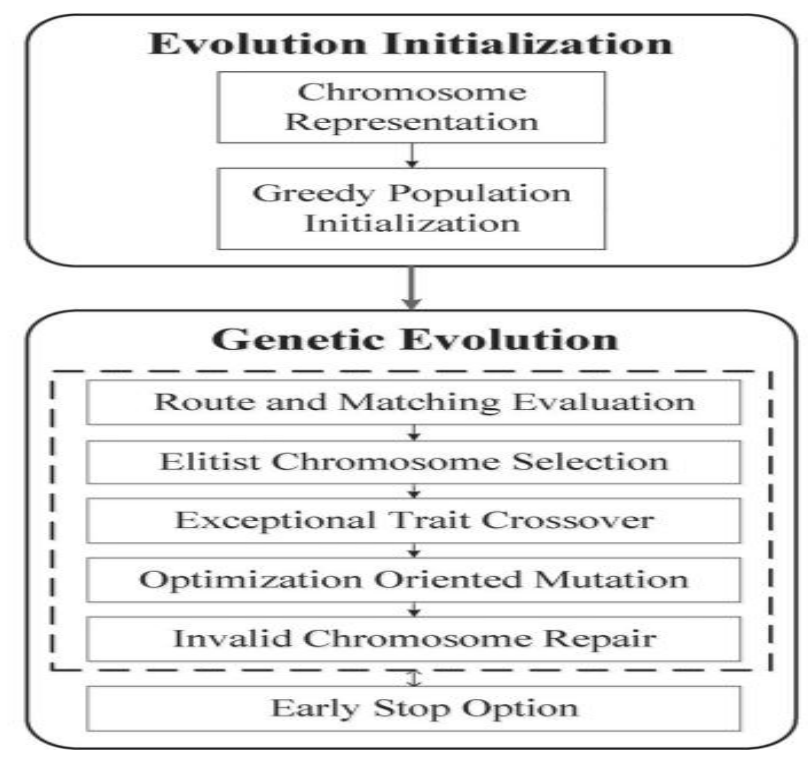

Figure 1.Proposed GCRMA

GCRMA determines carpool matches via the ICS and thereby provides an effective solution to the CSP. As shown in Fig.1, the GCRMA consists of two important modules: 1) an evolution initialization (EI) module and 2) a genetic evolution (GE) module. The EI stands for Evolution Initialization which consists of chromosome representation of user's request and feasible matches are then generated by the greedy population initialization procedure via distance-based heuristics. GE module consist of six proposed procedures: First, the route and matching evaluation takes place, after which elitist chromosome selection occurs. Then, exceptional trait crossover is implemented, followed by optimization-oriented mutation and invalid chromosome repair. Finally, the early stop option is employed. To obtain optimized solution the above procedure is repeated many times.

\subsection{Evolution Initialization Module}

5.1.1 Chromosome Representation

The request is represented as a chromosome

Req(ID,N,L,D,C,S)

Where,

ID: User ID;

N: Seat Number;

L: Current Location;

D: Destination;

C: Category - 0:Passenger\& 1:Driver;

S: Credit Score

which is input into the proposed GCRMA.

The carpool request includes $\mathrm{m}$ drivers and $\mathrm{n}$ passengers, which can be expressed as: 


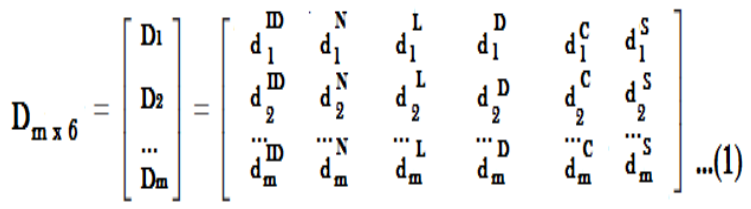

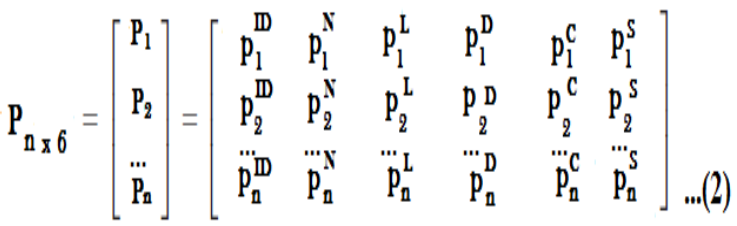

We propose a two-layer representational procedure, which is composed of 1) an assignment layer and 2) an implicit routing layer. This assignment layer can be divided into several segments, where each segment consist of a driver and passengers allocated to that driver, this design can effectively represent the number of drivers to whom passengers are allocated. Fig. 2 illustrates an example, in which Passenger 3, Passenger 8, and Passenger 30 are assigned to Driver1, and Passenger 22 and Passenger 18 are assigned to Driver 2.In the search space of the CSP, the number of segments can be dynamically modified to suit the quantity of drivers.

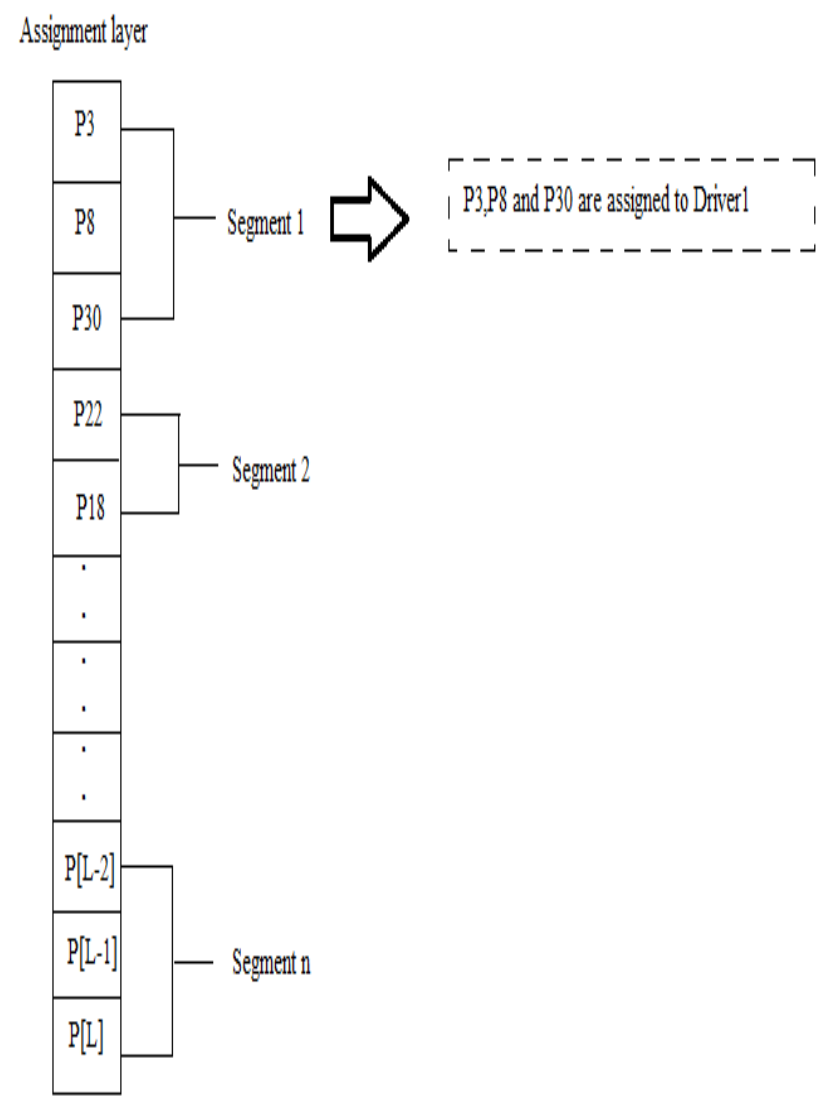

Figure 2.Assignment layer for proposed chromosome representation

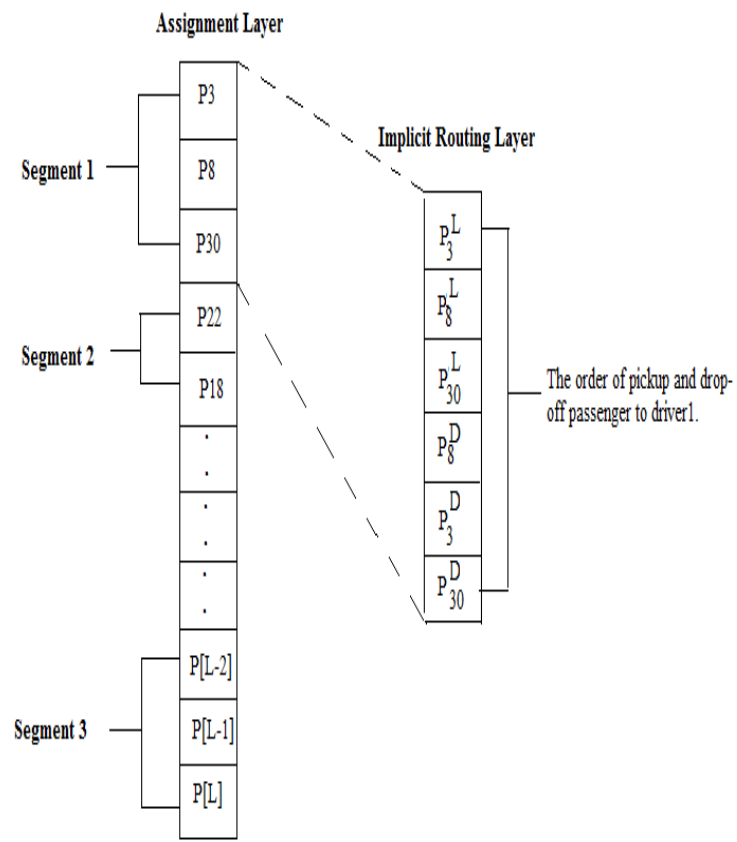

Figure.3.Implicit routing layer of proposed chromosome representation

The order in which drivers should pick up and drop off passengers is expressed by the implicit routing layer. Figure 2 presents an example in which Segment 1 features: a carpool consisting of Driver 1, Passenger 3, Passenger8, and Passenger 20. The implicit routing of Segment 1 shows that Driver1 will pick up passengers according to a specific order(Passenger3, Passenger8, and Passenger30) and drop off each passenger according a different order (Passenger8, Passenger3, and Passenger30)

\subsubsection{Greedy Population Initialization}

To effectively distribute the initial population in the solution space, the chromosomes that are first generation are arranged by designating a driver through the greedy strategy during the assignment process. The initialization procedure applies the proposed assignment strategy, called distance-based greedy heuristics.

Each driver is allocated passengers according to the magnitude of estimation values stored in set $\mathrm{EV}^{\mathrm{i}}=\left\{\operatorname{ev}^{\mathrm{i}}(\mathrm{j})\right\}$. The estimation value should be as minimum as possible and is expressed as:

$$
e v^{i}(j)=c\left(d_{i}^{L}, p_{j}^{L}\right)+c\left(p_{j}^{L}, p_{j}^{D}\right)+c\left(p_{j}^{D}, d_{i}^{D}\right)-c\left(d_{i}^{L}, d_{i}^{D}\right)
$$

where,

$\mathrm{c}(\mathrm{w}, \mathrm{x})$ is a travel distance cost value between two nodes,

$\mathrm{d}_{\mathrm{i}}^{\mathrm{L}}$ is origin of driver,

$\mathrm{d}_{\mathrm{i}}^{\mathrm{D}}$ is destination of driver,

$\mathrm{p}_{\mathrm{j}}{ }_{\mathrm{L}}$ is origin of passenger,

$\mathrm{p}_{\mathrm{j}} \mathrm{D}_{\mathrm{i}}$ is destination of passenger.

Value of ev close to zero indicates that the detour distance of the driver's route is slightly increased when the origindestination pair is added to redirect the initial route. 


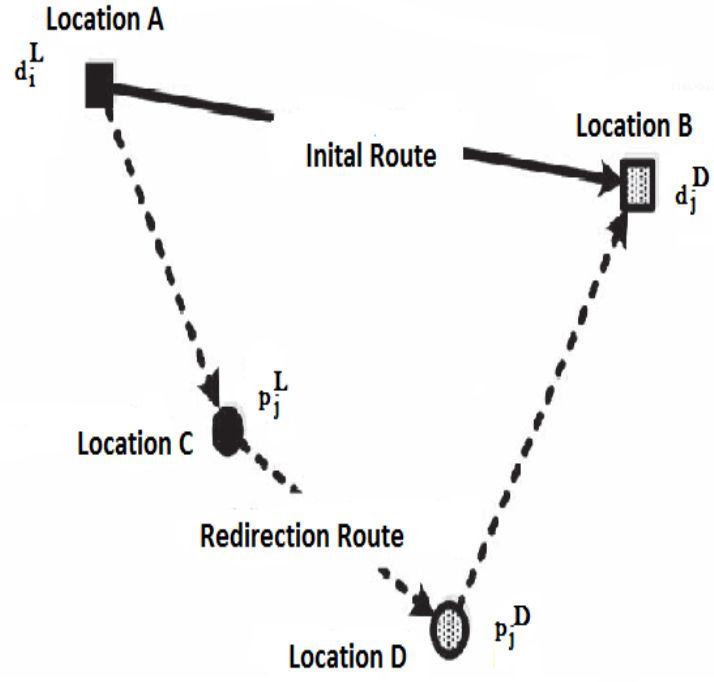

Figure 4. Change of route after adding origin-destination pair.

\subsection{Genetic Evolution Module}

\subsubsection{Route and Matching Evaluation}

The route and matching evaluation procedure finds the shortest route of each segment and subsequently records the routing results in the implicit routing layer of the chromosome. When the fitness value of chromosome is small,it indicates that better routing results are obtained. The fitness value of chromosome can be expressed as follows

$$
F_{k}=\Delta_{k}^{p}+\emptyset_{k}+\sum_{i=1}^{m} f i
$$

$\mathrm{F}_{\mathrm{k}}$ is the fitness value of kth chromosome; $\Delta_{k}^{p}$ represents the difference between total number of passengers and number of matched passengers and it should be 0 or greater than $0 ; \emptyset_{k}$ is reciprocal of sum total of all matches' rating; $f_{i}$ is the subfitness value of each segment.

The subfitness value of each segment can be calculated using subfitness function, given as follows

$$
\begin{gathered}
f_{i}=\left(C^{i}-\left|M_{i}\right|\right)+D T_{i} \ldots(5) \\
D T_{i}=\left\{\begin{array}{c}
\frac{2}{\pi} \cdot \tan ^{-1}\left(\text { dis }_{i}+\text { base }_{0}\right), i f\left|M_{i}\right|=0 \\
\frac{2}{\pi} \cdot \tan ^{-1}\left[d i s_{i}+\frac{\sum_{p \in M_{i}}\left(d i s_{p}^{w}+d i s_{p}^{t}\right)}{\left|M_{i}\right|}\right] \ldots(6)
\end{array}\right]
\end{gathered}
$$

Where $f_{i}$ is the subfitness of $i t h$ segment, $\operatorname{dis}_{\mathrm{i}}$ is the distance between the source and destination of driver, $d i s_{p}^{w}$ and $d i s_{p}^{t}$ are the waiting and travel distances respectively for each passenger allocated to driver $r_{i}, M_{i}$ is the set of passengers assigned to driver ${ }_{i}$, and base $_{0}$ is the average maximum distance travelled by passengers and it should satisfy base $e_{0} \gg>$ $\left(1 / M_{i}\right) \sum_{p \in M_{i}}\left(d i s_{p}^{w}+d i s_{p}^{t}\right) \forall i \in\{1,2, \ldots, m\}$.

To find the fitness value of each segment we need to find the most efficient route for picking up and dropping off passengers for each driver. The routing problem in a segment can be considered as a graph problem $\mathrm{G}=(\mathrm{N}, \mathrm{A})$, where $\mathrm{N}=\{\mathrm{n} 1, \mathrm{n} 2, . . \mathrm{n} 2 \mathrm{M}\}$ is the node set that represents the locations of passengers, and $A=\left\{(n 1, n 2), \ldots,\left(n_{w}, n_{x}\right)\right\}$ is the set of arcs subjected to the constraint $\mathrm{w} \neq \mathrm{x}, \forall \mathrm{n}_{\mathrm{w}} \in \mathrm{N}, \forall \mathrm{n}_{\mathrm{x}} \in \mathrm{N}$. The dynamic programming method is used to separate the routing problem into various recursive subproblems. The problem is modeled as the Bellman equation and is expressed as

$$
C^{A}\left(o_{t}\right)=\min _{n \in N\left(o_{t}\right)},\left\{C\left(o_{t-1}, n\right)+C^{A}\left(o_{t-1}\right)\right\} \ldots \text { (7) }
$$

Where $C^{A}\left(o_{t}\right)$ represents the thus far accumulated criteria cost at node $\mathrm{O}_{\mathrm{t}}$, t ranges from 1 to $2 \mathrm{M}$ ( $\mathrm{M}$ is the number of the served passengers). $N\left(o_{t}\right)$ is union set of sets $L\left(o_{t}\right)$ and $D\left(o_{t}\right)$ , where $L\left(o_{t}\right)$ includes the current location node of passengers assigned to Driver ${ }_{\mathrm{i}}$ and set $D\left(o_{t}\right)$ includesthe destination node of passengers assigned to Driver $\mathrm{i}_{\mathrm{i}} \mathrm{C}(\mathrm{w}, \mathrm{x})$ represents the criteria cost between node $\mathrm{w}$ and node $\mathrm{x}$ as shown in the following:

$$
C(w, x)=c(w, x)+c\left(x, d_{i}^{D}\right)-c\left(w, d_{i}^{D}\right) \ldots(
$$

The initial criteria $\operatorname{cost} C^{A}\left(o_{0}\right)$ is obtained by the formula:

$$
C^{A}\left(o_{0}\right)=\min _{n \in N\left(o_{0}\right)}\{I(n)\} \ldots
$$

Where I(n) represents the criteria cost starting from the initial location of driver to node $n$, and $N\left(o_{0}\right)$ is a node set that unified both $\operatorname{set} L\left(o_{0}\right)$ containing the current locations of all passengers assigned to Driver ${ }_{i}$ and empty set $D\left(o_{0}\right)$. The Bellman equation is solved by forward induction until that node set $\mathrm{N}$ is empty. The fitness value is used as a guide for the below chromosome selection procedure that is elitist chromosome selection.

\subsubsection{Elitist Chromosome Selection}

After the evaluation of population we sort the chromosomes in ascending order and then partition them into two subpopulation: top tier and low tier. The top-tier chromosomes are retained for the next generation, and the lower-tier chromosomes will be recombined with the top-tier chromosomes during the next procedure to produce the potential offspring.

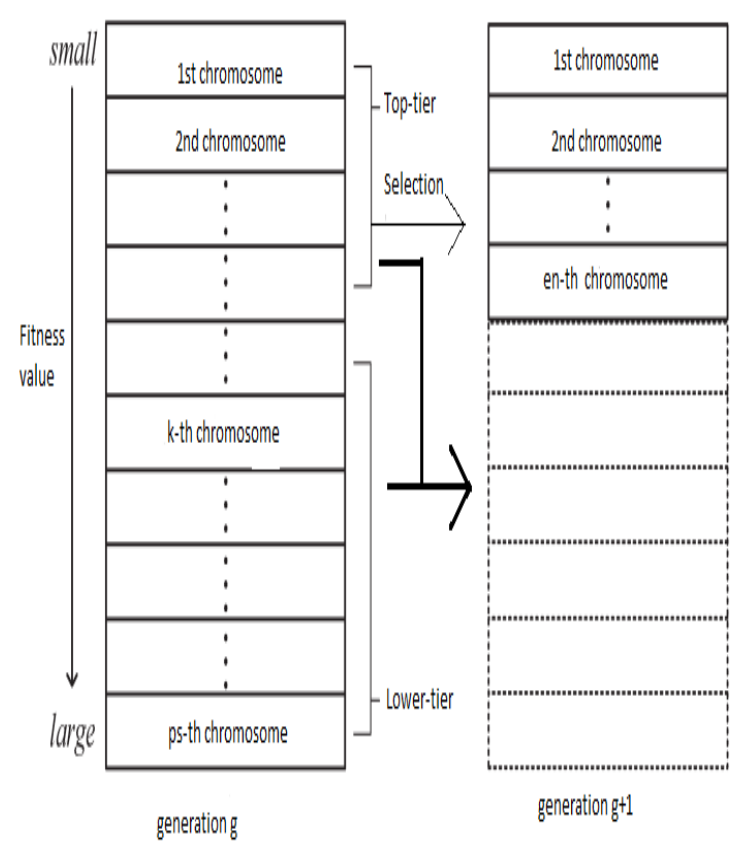

Figure 5.Chromosome selection of top tier and lower tier subpopulation 


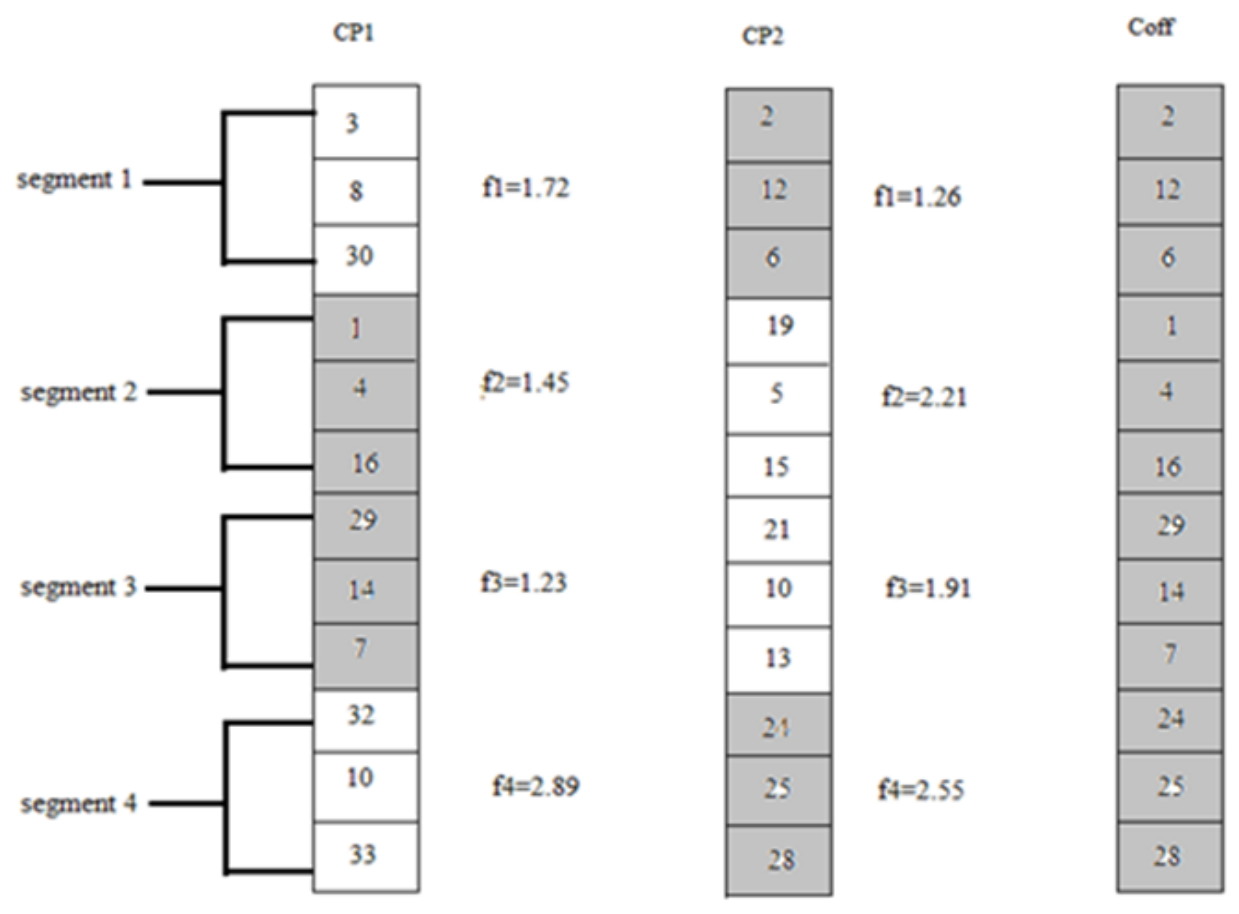

Figure 6.Elite-segment crossover procedure

\subsubsection{Exceptional Trait Crossover}

In exceptional trait crossover initial parent chromo-some

(Cp1) is randomly and uniformly selected from the top-tier chromosomes, second parent (Cp2) is selected from the lower-tier chromosomes to introduce mating diversity. Thus we obtain offspring chromosome (Coff) produced by recombination of $\mathrm{Cp} 1$ and $\mathrm{Cp} 2$. Here, let $\mathrm{m}$ represent the number of genetic segments; for $\mathrm{i}=1, \ldots, \mathrm{m}$, the ith segment of $\mathrm{C}_{\text {off }}$ inherits from either $\mathrm{Cp}_{1}$ or $\mathrm{Cp}_{2}$ according to the fitness value of the ith segment.

\subsubsection{Optimization Oriented Mutation}

To obtain optimized result there are two mutation operators: insert/reset and swap. Insert maximizes the number of matched passenger through insertion mutation. This insertion mutation begins at the segment that has the worst subfitness and performed by following functions

$$
\operatorname{seg}_{i}^{\prime}=\left\{\begin{array}{ll}
\operatorname{insert}\left(\operatorname{seg}_{i}\right), & \text { if }\left(C^{i}-\left|M_{i}\right|\right)>0 \\
\operatorname{reset}\left(\operatorname{seg}_{i}\right), & \text { if }\left(C^{i}-\left|M_{i}\right|\right)=0
\end{array}\right. \text {... }
$$

whereseg $_{i}$ is a mutated segment obtained from the segment $\operatorname{seg}_{\mathrm{i}}$ and insert operator is used to insert a new gene into the segment until its capacity is completely filled; otherwise, reset operator removes allgenes for reinsertion.

All passengers inserted into each segment are from the group of available passengers who have not yet been assigned to any driver.

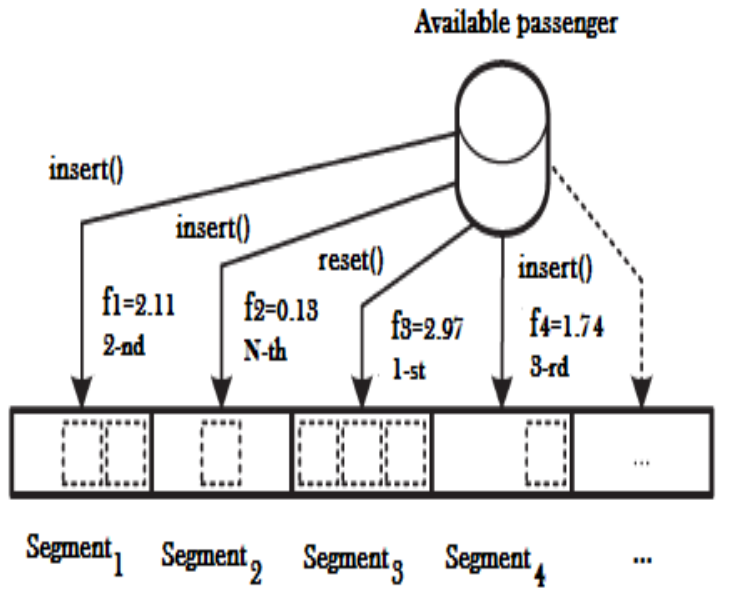

Figure7. Chromosome mutation of insertion operation

The second mutation operator is swap. According to the distance estimation value ev, the determinations of the mutation segments (e.g., sm1 and sm2) are selected from those segments assessed to have the worst fitness values. The multiple swap processes are performed by exchanging pair mutation points (e.g., pm1and pm2) that are selected from the pair of mutation segments until all poor-quality segments have 


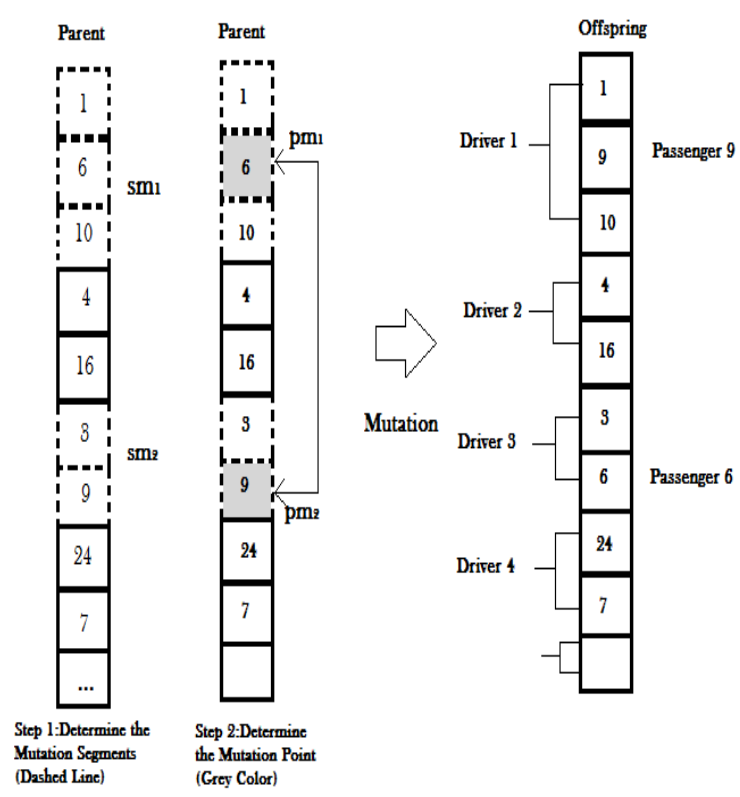

Figure 8. Chromosome mutation of swap operation

\subsubsection{Invalid Chromosome Repair}

In certain cases passenger could be assigned to more than one driver through above procedures and chromosome will become invalid. Hence it is necessary to replace repeated passengers with the passengers which have not yet been allocated to any driver. The capacity constraint can be satisfied by removing the excessive passengers to restore capacity validity.

\subsubsection{Early Stop Option}

An early stop option is based on the differences between the fitness values of consecutive generations and it reduces the computational cost of the GCRMA. There is point at which the evolution process reaches an optimum solution and should be terminated; this can be predicted by the relationship between fitness and generation via an accumulated variable $\left(\delta_{\mathrm{g}}\right)$, as

$$
\delta_{g}= \begin{cases}\delta_{g-1}+1, & \text { if } F_{B}^{g} \text { is equal to } F_{B}^{g-1} \\ 1, & \text { otherwise }\end{cases}
$$

Where $F_{B}^{g}$ is the fitness of the best solution in generation $\mathrm{g}$, and evolution will not be terminated until $\delta_{\mathrm{g}}>\mathrm{G}^{\mathrm{e}}\left(\mathrm{G}^{\mathrm{e}}\right.$ is an empirically selected threshold in the experimental results).

\section{CONCLUSION}

ICS provides an environment in which drivers and passengers can easily find carpool matches at any time and in any place via smart handled devices. GCRMA is genetic algorithm which is composed of two modules: an EI and a GE. EI consists of chromosome representation of user's request and uses distance-based greedy heuristics to generate initial population. The GE module is able to find the optimum carpool route and matching results, after which elitist chromosome selection occurs and exceptional trait crossover is implemented, followed by optimization-oriented mutation and invalid chromosome repair. The early stop option is additionally facility that provides the improvement of processing time.ICS comprises two primary modules: a mobile clients (MC) module and a carpool services (CS) module. The MC module is a mobile application built on an advanced mobile operating system such as Android. MC module, driver can offer carpool rides and passenger can send carpool requests. CS module provides the RESTful web services application interface for global implementation of the ICS on cross-platform devices. Thus carpooling is one of the most effective solutions to traffic congestion and thus reduces travel cost, energy consumption, and vehicle emissions.

In future, the above system can be modified to consider realtime traffic status. Thus, the route with lowest traffic congestion will be preferred by re-routing to low traffic roads. We can also take into account the pricing factor of using this system, which means every ride will be charged. This will make rides affordable to both driver and passengers. Moreover, to promote the concept of carpooling, we can also incorporate social networking like Facebook, Twitter, etc. This will also provide information of reputation of carpooling service providers.

\section{REFERENCES}

[1] B. T. Morris, C. Tran, G. Scora, M. M. Trivedi, and M. J. Barth,"Real-time video-based traffic measurement andvisualization system for energy/emissions," IEEETrans Intell. Transp. Syst. , vol. 13, no. 4, pp. 1667-1678, Dec. 2012.

[2] F.Terroso-Saenz, M. Valdes-Vela, C. SotomayorMartinez, R. Toledo-Moreo, and A. F. Gomez-Skarmeta, "A cooperative approach to traffic congestion detection with complex event processing and VANET," IEEE Trans. Intell. Transp. Syst., vol. 13, no. 2, pp. 914-929, Jun. 2012.

[3] V. Milanes, J. Godoy, J. Villagra, and J. Perez, "Automated on-ramp merg-ing system for congested traffic situations," IEEE Trans. Intell.Transp.Syst., vol. 12, no. 2, pp. 500-508, Jun. 2011.

[4] S. Hartwig and M. Buchmann, "Empty Seat Traveling," Nokia Research Center, Bochum, Germany, Feb. 2007.

[5] Son, Ta Anh, Le ThiHoaiAn, Pham Dinh Tao, and DjamelKhadraoui. "A Distributed Algorithm Solving Multiobjective Dynamic Carpooling Problem."International Conference onComputer \& Information Science.2012.

[6] Sghair, Manel, HayfaZgaya, Slim Hammandi, and Christian Tahon."A Distributed Dijkstra's Algorithm For The Implementation Of A Real Time Carpooling Service With An Optimized Aspect On Siblings." IEEE Annual Conference on Intelligent TransportationSystems.Madeira Island, Portugal, 2010.

[7] Guo, Yuhan - Goncalves, Gilles - Hsu, Tienté. "AMultiagent Based Self-adaptive GenerticAlgorithm for theLong-term Carpooling Problem." Springer Science Business Media B.V. 2012, 2011. 